\title{
Computational Examination of Aerodynamics Forces and Evolution of Vortex Shedding of Flow Past Three Square Cylinders at Two Symmetrical Vee Shapes
}

\author{
Salwa Fezai ${ }^{1 *}$, Fakher Oueslati ${ }^{2,3}$ and Brahim Ben-Beya ${ }^{3}$ \\ ${ }^{1}$ Physics Department, Samtah University College, Jazan University, 45142 Jazan, Kingdom of Saudi Arabia \\ ${ }^{2}$ Physics Department, Faculty of Science, Albaha University 6543 Al-Baha, Kingdom of Saudi Arabia \\ ${ }^{3}$ Laboratory of Physics of Fluids, Physics Department, Faculty of Science of Tunis, University of Tunis El-Manar, 2092 El-Manar 2, Tunis, Tunisia
}

*Corresponding author: Salwa FEZAI, Physics Department, Samtah University

College, Jazan University, 45142 Jazan, Kingdom of Saudi Arabia.

\begin{abstract}
The flow past three square cylinders at two symmetrical vee shapes has been investigated by a finite-volume method numerical technique. The two-dimensional computations have been performed for different Reynolds number (Re) ranging from 1 to 180 in order to consider different flow regimes; the steady, the periodic and the turbulent flow. The validation of the code with the available literature results regarded both the reliability of the computed solutions and the overall resulting efficiency of the methods. Velocity profiles, vorticity contours and integral parameters such as Strouhal number, drag and lift coefficients have been presented and analyzed. Steady and unsteady regimes of flow have been observed by monotonously changing the Re value, leading to the prediction of the critical Reynolds number for each configuration considered. Furthermore, the change in the geometry of the obstacle is found to affect dramatically the emergence of the Hopf bifurcation points. The oscillatory periodic wake is also seen to be influenced by the Strouhal number, which varies with obstacle shape and the Re values. Relevant outcomes of this work are the estimation of the vortex shedding frequencies and the prediction of the best configuration considering the computation of the lift and drag coefficients.
\end{abstract}

Keywords: Staggered square cylinders; Flow regimes; Vortex shedding; Strouhal number; Numerical simulation; Critical Reynolds number; Lift and drag coefficients

Nomenclature

(Nomenclature)

\begin{tabular}{|c|c|}
\hline Re & Reynolds number \\
\hline $\mathrm{l}$ & channel width \\
\hline $\mathrm{h}$ & channel height \\
\hline $\mathrm{c}, \mathrm{v})$ & velocity components, $\mathrm{m} \mathrm{s}^{-1}$ \\
\hline $\mathrm{x}, \mathrm{y}$ & dimensionless coordinates \\
\hline $\mathrm{St}$ & Strouhal number \\
\hline $\mathrm{CD}$ & drag coefficient \\
\hline $\mathrm{CL}$ & lift coefficient \\
\hline $\mathrm{p}$ & dimensionless pressure \\
\hline $\mathrm{f}$ & generic variable \\
\hline
\end{tabular}




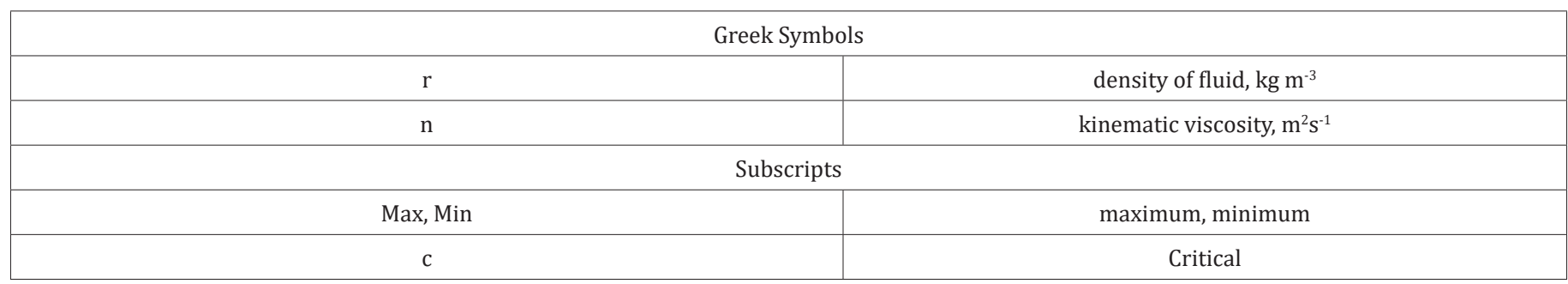

\section{Introduction}

The analysis of incompressible fluid flow around bluff bodies has engaged a particular attention of researchers due to its great importance in both aerodynamics and engineering. This type of flow becomes more and more complicated when single bluff body is replaced with multiple bluff bodies in different shapes and arrangements. This complexity in flow causes strong interactions in wakes and considerably modifies the fluctuation of aerodynamic forces.

Thus, it is important to understand the dramatic change in aerodynamics characteristics and evolution of vortex shedding frequency in the wake. In fact, many studies show that Reynolds number and the type of square cylinder arrangement has ability to change flow behavior for different flow states. In this vein, we may cite the work of Cheng et al. [1]. They have reported that the vortex shedding and wake development behind the square cylinder are significantly dependent on both the magnitude of the shear rate and the Reynolds number. One can also mention the numerical investigation of Berrone et al. [2]. Their results performed the influence of the Reynolds number on the appearance criteria of different regimes such as crawling scheme, the steady and unsteady with releases of vortices. In addition, Mukhopadhay et al. [3] analyzed the structure of a flow around a square obstacle for different Reynolds number and for different positions of the obstacle. They determined the critical Reynolds number from which the flow becomes periodic and they concluded that the frequency starts at $\mathrm{Re}=87$ for a blockage ratio $\mathrm{B} / \mathrm{H}=0.25$.

To understand the appearance criteria of steady regime, one can also refer to the investigation of Fezai et al. [4] who analyzed the vortex at different arrangements of the two shapes. Analysis of the flow evolution shows that with increasing Re beyond a certain critical value, the flow becomes unstable and undergoes a bifurcation. The authors demonstrated that the transition to unsteady regime is performed by a Hopf bifurcation and the critical Reynolds number beyond which the flow becomes unsteady is hence determined for each configuration. Lankadasu \& Vengadesan [5] observed that the critical Reynolds number is reduced with increasing shear. It was found that the mean drag coefficient decreases either with increasing shear for a particular Reynolds number or with increasing Reynolds number for a particular shear parameter. Sohankar \& Etminan [6] investigated the fluid flow around tandem square cylinders in laminar flow regime. Their results showed that the flow was steady when $\operatorname{Re} \leq 35$ and unsteady periodic when $\operatorname{Re} \geq 40$. Gera et al. [7] used the Computational Fluid Dynamics (CFD) technique to investigate the 2D steady flow around a square obstacle. The simulation was performed for flow around the square shape end to analyze the wake behavior and Reynolds number was taken from nearly 50 to 250. The coefficient of lift and velocity in the wake region were monitored to calculate the Strouhal number. Bhattacharyya \& Maiti [8] numerically analyzed the wake structure behind a square cylinder placed near the lower wall. They found that the wall causes a difference in strength between the two vortex rows and showed that the strength of the positive vortices from the bottom of the cylinder lowers with the decrease of the height between the barrier and the bottom wall. Subsequently, the average drag coefficient weakens with the reduction of the ratio of the standard height. They also proved that the cylinder has a large positive lift when brought close to the wall. The influence of the obstacle orientation has been analyzed by Sajjad \& Sohn [9]. They observed that orientation of a square cylinder has a significant effect on the shape and size of the recirculation bubble. They concluded that wake formation and separation processes are asymmetric for angles 22.5 and 30 degrees. In addition, a minimum in drag coefficient and maximum in Strouhal number is observed at a square cylinder orientation of 22.5. The main reason for the minimum drags coefficient at 22.5 is attributed to the wake asymmetry originated from shear layers of unequal lengths on each side of the cylinder. The wake asymmetry increases the transverse velocity, which further increases the base pressure, and hence lowers drag. This factor (wake asymmetry) is counterbalanced by an increase in the projected area. However, it was revealed that the former has an overall stronger influence at small cylinder angles shown by the drag coefficient minimum at an orientation of 22.5. Furthermore, they observed that the separation distance between the alternating vortices depend upon the cylinder orientation. On another hand, it is known in literature that the flow structure around two square cylinders is more complex than that around a single square cylinder. For instance, Burattimi \& Agrawal [10] analyzed the flow around two square cylinders at a Reynolds number of 73 and reported different ranges of the wake flow regimes. Abbasi \& Islam [11] also simulated wake interactions of a flow around two square cylinders that are placed in line with a fixed space ratio equal to 3.5. They concluded that the unsteady regime appears when the Reynolds number reaches the value 55.

Rao et al. [12] investigated the effect of the space ratio on the flow around two square cylinders arranged side by side. They found that the frequency of vortex shedding is different in two 
wakes. The upper frequency is smaller than the lower frequency for small rations $(s<1.4)$. However, when the space rations increase, the frequency of vortex shedding is almost equal in two wakes. They analyzed the influence of the space ratio and Reynolds number on the drag and lift force. The difference of time-averaged drag and lift coefficients of the cylinders decreases with the increase in space ratios. When $s=2.0$ and 2.5 , the curves for the time-averaged drag and lift coefficient with different Reynolds numbers are seen to be smooth. The authors also reported that when s=1.5 and 1.8, the curves are smooth under $\operatorname{Re}<140$, but will be fluctuant under Re $>140$ because of the nonlinear interaction between the wakes and the instability of flow which becomes stronger with the increase in Reynolds numbers. Furthermore, Adeeb et al. [13] investigated computationally the wake flow of two square cylinders by varying the corner radius to understand the effect of the gap spacing's role and the wake flow pattern at $\mathrm{Re}=100$. They found that the flow characteristics and vortex shedding depend significantly on the corner radius and gap spacing. They concluded that a square cylinder exhibited the maximum average drag value and an inverse behavior was observed in the case of a circular cylinder. In addition, aerodynamic forces were reduced by rounding the corner radius. The staggered geometry of different square cylinders is much different from tandem and inline configurations because more than one spacing is introduced between the cylinders. In such case the flow characteristics become different from those in the case of single spacing. Compared to tandem and inline arrangement, the staggered arrangement has received very less attention of analysis. Among the authors who analyzed this type of arrangement, one can cite Lee \& Yang [14]. The authors investigated the flow around two cylinders for $\operatorname{Re} \leq 160$. At staggered orientation of $45^{\circ}$ and $\operatorname{Re}=160$, they identified four different flow regimes. They reported that gap flow can induce separation on the downstream cylinder which asa result increases the vortex shedding phenomena. Aboueian \& Sohankar [15] examined the effect of the gap spacing on the flow over two square cylinders in staggered arrangement at $\mathrm{Re}=150$. By changing the gap spacing between cylinders, five different flow regimes are identified and classified. Islam et al. [16,17] examined the influence of separation rations and Reynolds number for flow around squares cylinders. They found that the flow structure depends on both separation ratio and Reynolds number. The average mean drag coefficient decreases while Strouhal number increases as the gap ratio $(\mathrm{g})$ increases. They observed that as this parameter value increases the average mean drag coefficient and Strouhal number approaches to single rectangular cylinder value.

The flow behind three square cylinders in two different triangular arrangements was investigated numerically by Rahman et al. [18]. They reported that the Strouhal number varied between 0.0132 and 0.2003 in both arrangements. The authors also found that the mean drag coefficient and Strouhal number of all threesquare cylinders approached single square cylinder with the increase in the gap ratio. Yang et al. [19] analyzed numerically the flow pattern and vortex suppression regions for three circular cylinders with two different staggered arrangements. In the first arrangement a primary cylinder was placed in front of downstream two side by side cylinders and in the second arrangement a primary cylinder was placed in the wake of upstream two side by side cylinders. Their results show that in the first arrangement, the vortex shedding behind the primary cylinder was suppressed by the downstream two side by side cylinders at $100 \leq \mathrm{Re} \leq 200$. They also reported that in the second arrangement the vortex shedding of the primary cylinder was suppressed by the upstream two side by side cylinders at $103 \leq \operatorname{Re} \leq 175$. We can also mention the work of Yang et al. [20] who arranged three stationary circular cylinders in such a way that one cylinder was placed in front of two side by side cylinders and investigated the effect of the gap ratio in the range from $\mathrm{g}=1-10$ for $\mathrm{Re}=200$. They observed steady flow region at $1 \leq \mathrm{g} \leq 1.2$ and $2.5 \leq \mathrm{g} \leq 3.1$ and unsteady flow region at $1.3 \leq \mathrm{g} \leq 2.4$ and $3.2 \leq \mathrm{g} \leq 10$. They also found regular drag and lift coefficient in steady flow region which became irregular in unsteady flow region. From the previous studies about fluid interaction with solid bodies, it can be observed that there is very limited numerical work available about the flow mode transitions around three staggered square cylinders at low values of Re. The flow around bluff bodies of square cross section is an important fundamental problem of engineering. Also, the staggered square cylinders geometry is vital in the sense that it is very important in practical engineering applications. Therefore, a detailed analysis of flow past three staggered square cylinders is needed. Furthermore, this study will definitely enhance the fluid-structure interaction database. Keeping in view these facts the current numerical study is performed to fully analyze four important features of the flow past three staggered square cylinders in vee shape:

The first task is essentially composed of six parts. Firstly, we present the configuration of the physical problem. The second part is devoted to presentation of equations and boundary conditions. The numerical method used for solving the system of Navier-Stokes equations in its dimensionless form in the case of a two-dimensional (2D) incompressible flow is presented in the third part. The fourth part is dedicated to the validation of the numerical in-house code used and followed finally by results and discussions. The fifth part is devoted to results and discussions. Finally, we will draw conclusions from this study in a final part.

\section{Physical Problem}

(Figure 1) The configuration of the current problem is sketched in Figure 1. Two configurations will be considered by varying the arrangement of the three cylinders C1, C2 and C3. For the first configuration the obstacle is composed of three cylinders C1, C2 and C3 as shown in the figure. For the second configuration the arrangement of the three cylinders will be different from the first configuration. In Figure 2 we have schematized the two configurations that will be studied and examined (Figure 2). 


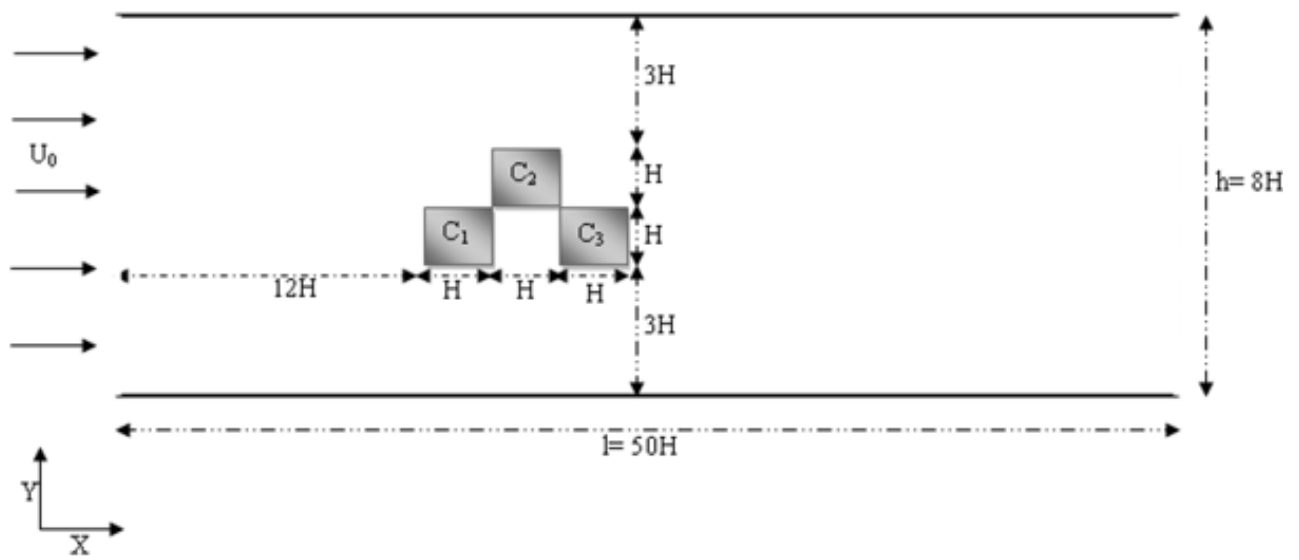

Figure 1: Physical model.

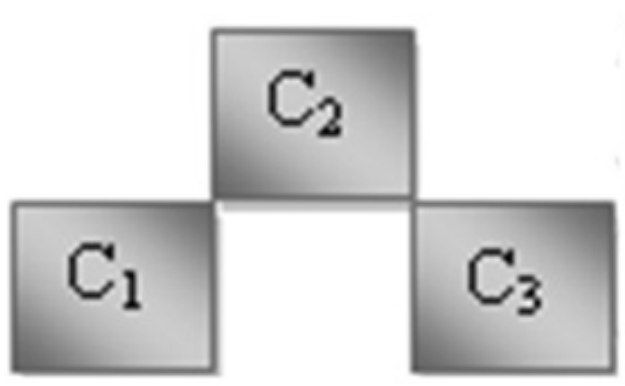

Configuration 1

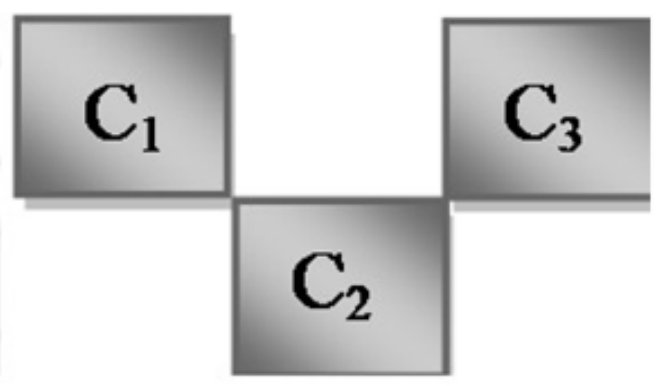

Configuration 2

Figure 2: Sketch of the two configurations considered in this study.

\section{Governing flow equations}

The physical problem is governed by the Navier-Stokes equations for two-dimensional incompressible fluid flow. The continuity and momentum equations are written in dimensionless form expressed as follows:

$$
\begin{gathered}
\frac{\partial u_{i}}{\partial x_{i}}=0 \\
\frac{\partial u_{i}}{\partial_{t}}+\frac{\partial\left(u_{i} u_{j}\right)}{\partial x_{j}}=-\frac{\partial p}{\partial x_{i}}+\frac{1}{R_{e}} \frac{\partial^{2} u_{i}}{\partial x_{j}^{2}}(2)
\end{gathered}
$$

Generally, a dimensionless writing of the Navier-Stokes equations shows the usual dimensionless numbers. These parameters characterize the similarity structure and stability of the flow. It is

worth noting that the scales $\mathrm{H}, \mathrm{u}_{0}, P_{o}=\frac{1}{2} \rho_{o} u_{o}^{2}$ and $t_{o}=\frac{H}{u_{o}}$ are used for the dimensionalization of the coordinate space $\mathrm{x}_{\mathrm{i}}=(\mathrm{x}, \mathrm{y})$, velocity $\mathrm{u}_{\mathrm{i}}=(\mathrm{u}, \mathrm{v})$, pressure $\mathrm{p}$ and time $\mathrm{t}$ respectively.
In the momentum equation, a dimensionless number appears that is the Reynolds number

expressed by:

$$
\operatorname{Re}=\frac{u_{e} H}{v}
$$

Where $u_{e}=u_{o}$ is the velocity upstream, $\mathrm{H}$ is the side of the barrier andn is the kinematic viscosity of the fluid under consideration.

\section{Boundary Conditions}

The boundary conditions for this physical problem are as following:

1. At the channel entrance:

1. The horizontal $\mathrm{u}$ - velocity component has a uniform form $\mathrm{u}_{0}=1$ The vertical component of the velocity $\mathrm{v}$ is set to zero.

2. On the obstacle, non-slip conditions are imposed; $u=0$ and $v$ $=0$ 
3. At the exit of the channel: The convective condition [21] is written as follows:

$$
\text { 2. } \frac{\partial u_{i}}{\partial_{t}}+u_{\text {conv }} \frac{\partial u_{i}}{\partial x}=0 \text { where, } u_{\text {conv }}=u_{e}=1
$$

4. At the upper and lower walls: The far field boundaries are defined as slip wall with

$$
\frac{\partial u}{\partial y}=0 \text { and } v=0
$$

\section{Numerical Method}

The dimensionless Navier-Stokes equations were numerically solved utilizing the following numerical technique based on the finite volume method [22]. The temporal discretization of the time derivative is performed by an Euler backward second-order implicit scheme. Nonlinear terms are evaluated explicitly; then, viscous terms are treated implicitly. The strong velocity-pressure coupling present in the continuity and the momentum equations is handled by applying the projection method [23]. A Poisson equation, with the divergence of the intermediate velocity field as the source term, is then computed to obtain the pressure correction and the real velocity field. A finite volume method was utilized on a staggered grid system in order to discretize the system of equations to be solved. Furthermore, the QUICK scheme of Hayase et al. [24] is applied to minimize the numerical diffusion for the advective terms. The discretized equations are computed utilizing the red and black point successive over-relaxation method [25] with the choice of optimum relaxation factors. Besides, the resolution of the Poisson equation is performed by applying an accelerated full multi-grid method [26].

The convergence of the numerical results is established at each time step according to the following criterion:

$\sum\left|\phi_{i, j}^{\tau}-\phi_{i, j}^{\tau-1}\right|<10^{-8}$

Where $f$ and $t$ indicates the iteration, time levels. The generic variable $f$ stands for $\mathrm{u}, \mathrm{v}$ or $\mathrm{p}$. In the above inequality, the subscript sequence $(\mathrm{i}, \mathrm{j})$ stands for the grid indexing in the $\mathrm{x}$, and $\mathrm{y}$ directions respectively. In conclusion, it is worth noting that computations were performed by applying a developed home code named "NASIM" (Navier Stokes Incompressible Multigrid) [27,28] utilizing fi- nite volume method and the numerical procedure described above.

\section{Results and Discussion}

\section{Grid independence test}

The construction of the mesh is the first step in any numerical simulation. The used meshes in this investigation are based on a staggered grid where scalar quantities (pressure ...) are located at the center of the cell, while the velocity components are defined at the centers of faces of the volume controls. In order to ensure accuracy, the grids have denser clustering at the vicinity of the barrier where strong gradients are expected. Conversely, away from the obstacle where the expected gradients are low, larger meshes are preferred.

It is worth noting that the grid independence study was conducted for two different non- uniform grids, namely, $m * n=560 * 340$ and $m^{*} n=768 * 160$. In the current investigation, independence of numerical results from the mesh size was assumed when the difference in the simulated values computed between two consecutive grids was less than $1 \%$. It was concluded from the deviation values obtained that, a non-uniform grid of $m^{*} n=768^{*} 160$ is sufficiently fine to ensure the grid independent solution and provides a good compromise between accuracy and CPU time in the range of variables to be investigated. Hence, the grid $m^{*} n=768^{*} 160$ is applied to perform all subsequent calculations. For better clarification, Figure 3 is schematized describing the mesh used in the present simulations (Figure 3).

\section{Time step independence test}

To ensure the accuracy of the results, it is necessary to verify the numerical procedure. The determination of the time step to be utilized in all calculations is very important, since a too large time step size will yield inaccurate results while an excessively smalltime step is computationally inefficient. Furthermore, several time step refinements were performed for the present simulations with non-uniform grid $m^{*} n=768 * 160$. To ensure the temporal convergence of results, two Figures $4 \& 5$ are reported describing the temporal evolution of the lift coefficient and horizontal velocity components, respectively (Figures 4,5).

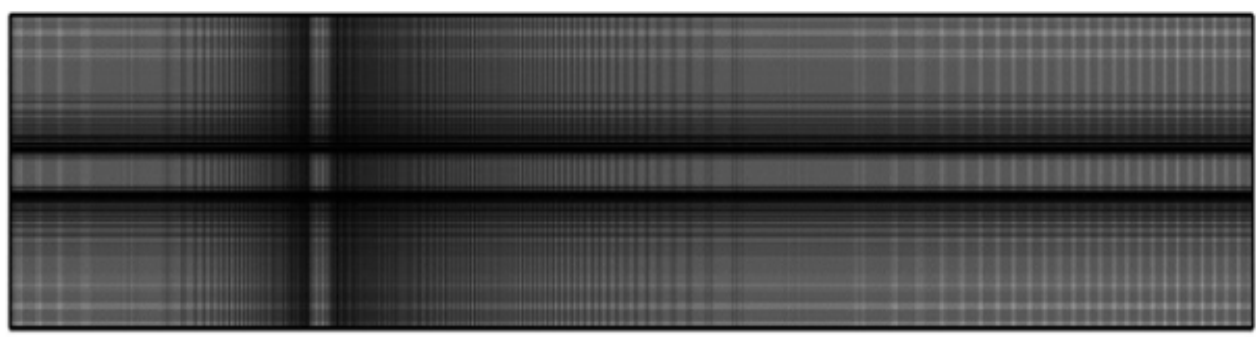

Figure 3: Non-uniform mesh of dimension $m^{*} n=768^{*} 160$. 


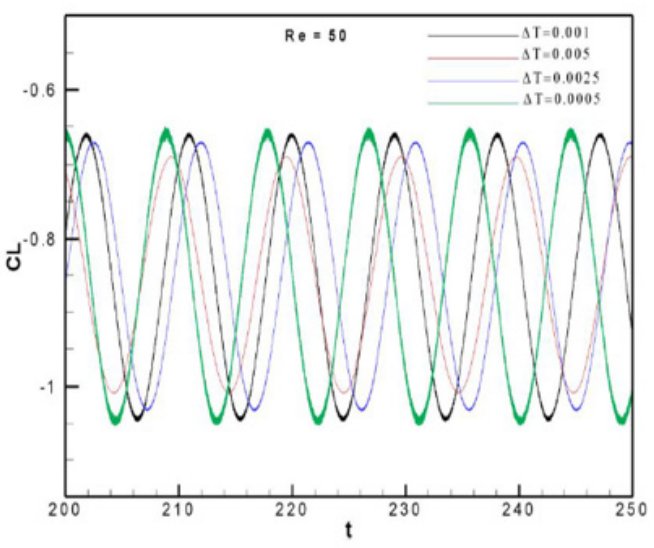

Figure 4: Temporal evolution of the lift coefficient for different time step.

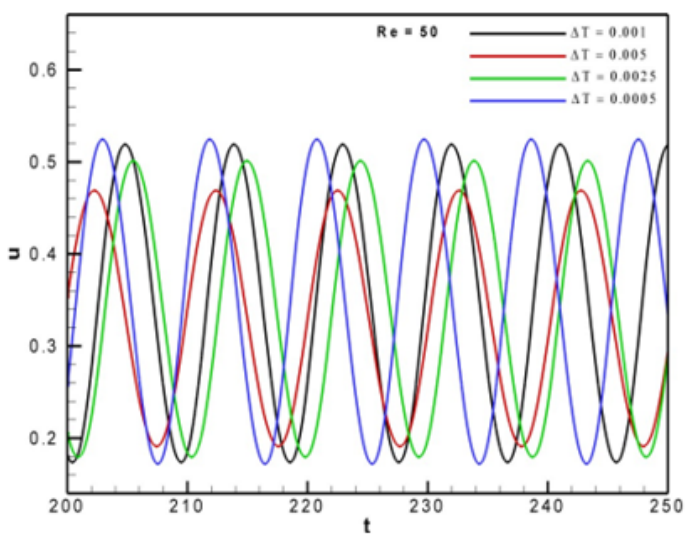

Figure 5: Temporal evolution of the horizontal velocity component for different time step.

From Table 1, it is confirmed that the results of the calculations with the time step $\Delta t=0.001$ are nearly identical to the results with $\Delta \mathrm{t}=0.0025$ and $\Delta \mathrm{t}=0.0005$. That's why the time step $\Delta \mathrm{t}=0.001$ is applied in all simulations (Table 1 ).

\section{Code validation}

To give more confidence to the results of the current simulations, some quantitative and qualitative comparisons with other numerical investigations presented in the literature have been carried out. The physical problem that is used for the validation of the in-house code "NASIM" is the physical model investigated by Breuer et al. [29]. In the current study, validation of the simulations was carried out on a non-uniform mesh of dimension $m^{*} n=560 * 340$ as the mesh used by Breuer et al. [29]. The study was conducted over a range of Reynolds number varying from 10 to 180 and analysis of the effect of this parameter on the evolution of the Strouhal number and lift and drag coefficients was carried out. Consequently, the effect of Re on the Strouhal number (Figure 6) was performed. It is noted that for relatively low Reynolds numbers $(50<\operatorname{Re}<130)$ the Strouhal number increases with Re values. A significant change in the structure of flow takes place, namely the movement of separation point of the trailing edge to the leading edge of the square cylinder. The Strouhal number is at a maximum at nearly $\mathrm{Re}=140$ then decreases again for higher Reynolds numbers (Figure 6).

The resulting physical parameter like St obtained from these computations is presented in table 2 along with percentage deviation in the code verification with Ref. [29] and Ref. [30]. It is clear from the results that deviation percentage with Ref. [30] is weaker with that of Ref. [29]. Note that the maximum deviation percentage with Ref. [29] and Ref. [30] is respectively 9.69\% (when $\mathrm{Re}=200$ ) and $2.91 \%$ (when $\mathrm{Re}=180$ ). In fact, this percentage of maximum deviation has been obtained for the large Reynolds numbers and this leads to the weak refinement of the computing grid. In other words, in order to minimize the error, it is necessary to increase and further refine the mesh for large Reynolds values. In fact, by further

incrementing the Reynolds number, more instabilities of the flow may occur, and this may cause the appearance of more vortices and small structures, and for a better visualization of the flow pattern and to capture its structures, the mesh must be further refined (Table 2).

As observed, Figure 5 and Table 2 confirm that our results are in good agreement with the results of Breuer et al. [29] \& Galleti et al. [30] and this comparison validates our computer code making 
confidence on the presented results. It is also worth noting that validation of the computer code has already been presented by Fezai et al. $[31,32]$ which is a contribution of the current investigation.

\section{Analysis of the evolution of vortex shedding of flow}

\section{Steady regime}

In the previous section, the code validation was discussed. More detailed flow pattern, which is greatly dependent on the geometry of the obstacle, is further investigated in Figure 7 where vorticity contours at $\mathrm{Re}=1$ relatively to the considered configurations are presented. It is clear that the flow is creeping and the viscous force being preponderant. It can be seen that the fluid remains attached to the obstacle for each configuration and no detachment is observed. Thus, we note the symmetry of the flow with respect to the longitudinal axis. The flow is formed by two almost symmetrical counter-rotating recirculating lobes attached to the obstacle. It is confirmed that the geometry of the obstacle did not modify the structure of the flow since both configurations have the same struc- ture (Figure 7).

The intensity of the viscous force is reduced by augmenting Re to a certain value, during which the separation of the laminar boundary layers occurs. Figure 8 depicts the contours of the iso-vorticities of the two configurations considered for $\mathrm{Re}=10$ and 20. It is observed that the wakes of the two configurations are similar. As displayed in the figure, the state of the flow remains steady. The flow is always symmetrical with respect to the longitudinal axis but loses its symmetry with respect to the upstream and the downstream. A wake appears formed by two symmetrical counter-rotating recirculating lobes attached to the obstacle and occupying the whole volume. Due to the increase of Re, the forces of inertia strengthen and prevent the boundary layer to remain attached to the obstacle and starts to favor a depression in the wake zone. We may note the beginning of birth of the vortex that develops far from the obstacle in the second configuration when $\mathrm{Re}=20$ because one approaches the bifurcation point towards the unsteady regime (Figure 8).

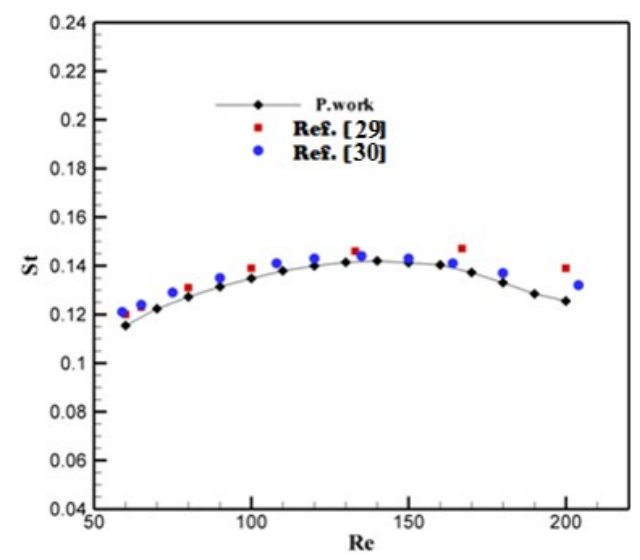

Figure 6: Variation of the Strouhal values versus the Reynolds number.

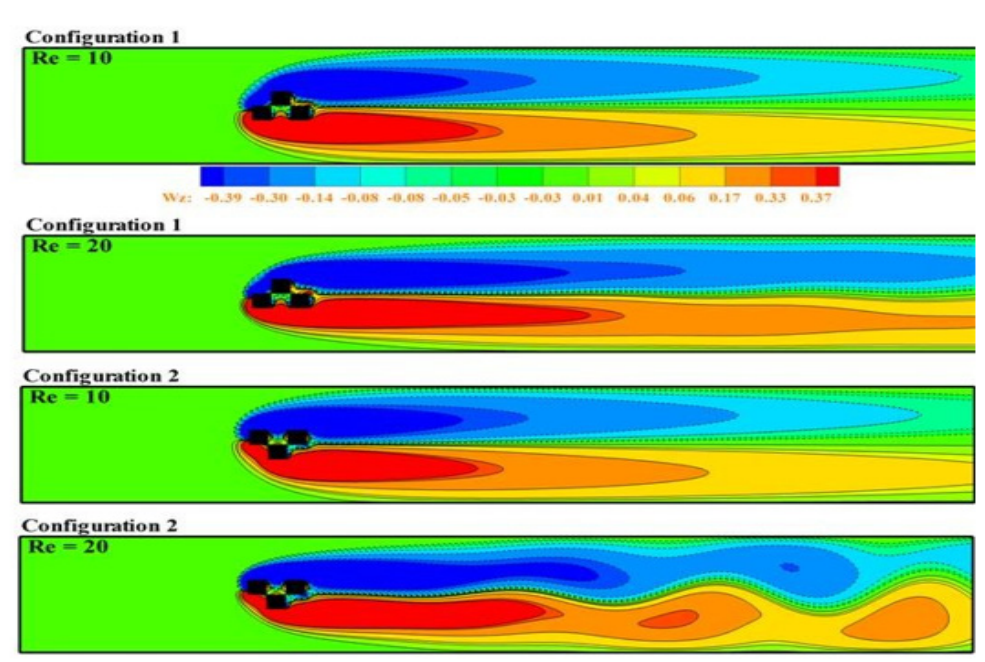

Figure 8: Vorticity contours at $\mathrm{Re}=10$ and 20 relatively to the two considered configurations. 


\section{Hopf Bifurcation point}

In this section the hop bifurcation point will be determined and discussed. It can be concluded that the flow is oscillatory from a certain Reynolds value. This periodic behavior occurs when the Reynolds number exceeds the critical value (Rec). It should be noted that the critical value of Re strongly depends on the geometry of the obstacle. Indeed, we consider performing several simulations for several Reynolds for both considered configurations. In order to compute the critical Reynolds number for each configuration, we start from $\mathrm{Re}=20$ and enhance it with the increment of 0.5 until we find the critical value. From the time when the perturbations change in an oscillatory trend, the flow after the critical point remains periodic in time and undergoes a critical value of Reynolds number. Besides, the square root of the amplitude of the solution increases with the bifurcation parameter. This means that the square of the amplitude of $u$ and v velocity components must be proportional to the value of Re after the bifurcation. It should be noted that the amplitude of the velocity component ( $u$ or $v$ ) is defined

as: $A m p=\left|u_{\max }(A, t)-u_{\text {min }}(A, t)\right| / 2$, where the coordinate point A $(16 ; 4.4)$ is taken in the wake of the obstacle. During this procedure, we noted progressively the amplitude squared values $\mathrm{Amp}^{2}$ of the velocity components $\mathrm{u}(\mathrm{A})$ and $v(\mathrm{~A})$. This allowed us to plot the amplitudes squared of the oscillations $\left(\mathrm{Amp}^{2}\right)$ as a function of the Reynolds number in Figure 10.

Table 1: Influence of time step $(\Delta t)$ at $\operatorname{Re}=50$.

Table 1: Influence of time step $(\Delta \mathrm{t})$ at $\mathrm{Re}=50$.
\begin{tabular}{|c|c|c|c|}
\hline Dimensionless Time Step $(\Delta \mathrm{T})$ & Strouhal Number (St) & Time Averaged Drag Coefficient & 3.0331 \\
\hline 0.005 & 0.0986 & 3.0334 & 0.2603 \\
\hline 0.0025 & 0.1057 & 3.033 & 0.2939 \\
\hline 0.001 & 0.1101 & 3.0331 & 0.3078 \\
\hline 0.0005 & 0.112 & 0.3131 & \\
\hline
\end{tabular}

Table 2: Percentage deviations in the code verification with Ref. [29] and Ref. [30].

\begin{tabular}{|c|c|c|c|c|c|}
\hline Reynolds Number & Present Work & Ref. [29] & Percentage Deviations with Ref. [29] & Ref. [30] & Percentage Deviations with Ref. [30] \\
\hline 60 & 0.11543 & 0.12 & $3.81 \%$ & ----- & ---- \\
\hline 70 & 0.1223 & $-\cdots$ & $-\cdots$ & $-\cdots-$ & ---- \\
\hline 80 & 0.1272 & 0.131 & $2.90 \%$ & ---- & ---- \\
\hline 90 & 0.1314 & ----. & ----- & 0.135 & $2.66 \%$ \\
\hline 100 & 0.13477 & 0.139 & $3.04 \%$ & ---- & ---- \\
\hline 110 & 0.13786 & ---- & ---- & ---- & ---- \\
\hline 120 & 0.13996 & ---- & ---- & 0.143 & $2.13 \%$ \\
\hline 130 & 0.14142 & ---- & ---- & ---- & ---- \\
\hline 140 & 0.14201 & ---- & ---- & ---- & ---- \\
\hline 150 & 0.1412 & ---- & ---- & 0.143 & $1.25 \%$ \\
\hline 160 & 0.1403 & ---- & ---- & ---- & ---- \\
\hline 170 & 0.1372 & ---- & ---- & ---- & ---- \\
\hline 180 & 0.133 & ---- & ----- & 0.137 & $2.91 \%$ \\
\hline
\end{tabular}




\begin{tabular}{|l|c|c|c|c|c|}
\hline 190 & 0.1284 & $-\cdots--$ & $-\cdots---$ & $-\cdots$ \\
\hline 200 & 0.12553 & 0.139 & $9.69 \%$ & $\cdots---$ & $\cdots$ \\
\hline
\end{tabular}

Table 3: Comparison of results for the flow past a single square cylinder in terms of percentage reduction.

\begin{tabular}{|c|c|c|}
\hline & Configuration 1 & Configuration 2 \\
\hline $\mathrm{Re}_{c}$ & 22.45 & 22.37 \\
\hline Percentage reduction & $57.64 \%$ & $57.79 \%$ \\
\hline
\end{tabular}

Table 4: Comparison of results for the flow past a single square cylinder in terms of percentage reduction.

\begin{tabular}{|c|c|c|c|c|c|}
\hline Re & St (square cylinder) & St (Configuration 1) & \%Reduction & St (Configuration 2) & \%Reduction \\
\hline 60 & 0.11543 & 0.10327 & 10.54 & 0.10262 & 11.1 \\
\hline 80 & 0.1272 & 0.10574 & 16.87 & 0.10559 & 16.99 \\
\hline 100 & 0.13477 & 0.10597 & 21.37 & 0.10667 & 20.85 \\
\hline 120 & 0.13996 & 0.10386 & 25.79 & 0.10486 & 25.08 \\
\hline 140 & 0.14201 & 0.10068 & 29.1 & 0.10262 & 27.74 \\
\hline 160 & 0.1403 & 0.09974 & 28.91 & 0.10116 & 27.9 \\
\hline 180 & 0.133 & 0.09845 & 25.98 & 0.10098 & 24.08 \\
\hline
\end{tabular}

Table 5: Mean values of the drag coefficient of both configurations for different Reynolds numbers.

\begin{tabular}{|c|c|c|}
\hline Re & CD (Configuration 1) & CD (Configuration 2) \\
\hline 1 & 70.8319 & 10.0823 \\
\hline 10 & 10.9456 .1038 \\
\hline 20 & 7.15196 & 6.88916 \\
\hline 30 & 5.81073 & 5.73654 \\
\hline 40 & 5.19232 & 5.14265 \\
\hline 60 & 4.5323 & 4.58401 \\
\hline 80 & 4.2487 & 4.35963 \\
\hline 100 & 4.14623 & 4.3031 \\
\hline 120 & 4.14636 & 4.34456 \\
\hline 140 & 4.22776 & 4.36158 \\
\hline 160 & 4.31928 & 4.54506 \\
\hline 180 & 4.41261 & 4.55154 \\
\hline
\end{tabular}

\section{Unsteady regime}

As the Reynolds number exceeds the critical value, the flow becomes unsteady. At this stage, the instabilities are triggered which generates a noticeable change in the flow structure. To better analyze this change, Figure 10 is presented illustrating the effect of the Reynolds number on the iso-vorticities of the two configurations. For low Reynolds numbers ( $\operatorname{Re}=40$ and 60$)$, it is noted that the structure of the flow is similar in both configurations. A pair of vortices appears with opposing signs alternately separating behind each obstacle. In fact, the influence of the viscous force becomes negligible. However, the intensity of the inertial force strengthens with Reynolds increase so this force becomes dominant knowing that this number is defined as the ratio of the inertia force by the viscous force. As the Reynolds number augments, the shape and size of the vortices change in both configurations. This change is due to the influence of the obstacle geometry on the vortex structure. Therefore, we note that in the first configuration the shape of the vortices of negative sign becomes very elongated and these swirls dominate the wake, but the vortices of positive sign keep the round shape. Due to the lengthening of the negative sign vortices, the lateral and longitudinal spacing increases in the wake so this spacing causes a significant change in the fluctuation of the pressure. On the other hand, in the second configuration, the shape of the vortices of negative sign remains round but a significant change establishes in the shape of the vortices of positive sign which makes these vortices very elongated. The intensity of these positive vortices dominates and increases while increasing the Reynolds number. It can also be seen that the interaction between vortices of different intensities leads to a vortex structure proportional to the shape of the obstacle with a lateral and longitudinal spacing as proportional to the geometry of the obstacle.

It is clear that the flow pattern depends strongly on the shape of the obstacle. It can be deduced then that the two configurations generate a significant change in the characteristics of the wake be- 
cause the lengthening of these vortices causes a remarkable spacing in the wake. Therefore, the spacing in the wake leads to diminishing the pressure and enhancing the speed. In order to verify the fluctuation of the velocity in the wake of both configurations, the variation of the horizontal component $(\mathrm{u})$ of the velocity as a function of the Reynolds number is shown in Figure11. It can be seen that the velocity variation in both configurations increases mono- tonically as a function of the Reynolds number. However, it should be noted that the variation of the speed is very high in the second configuration and the smallest fluctuation of $(\mathrm{u})$ is noted in the first configuration. It can be deduced that the second configuration causes a sharp decrease in the variation of the pressure compared with the first configuration (Figures 10,11).
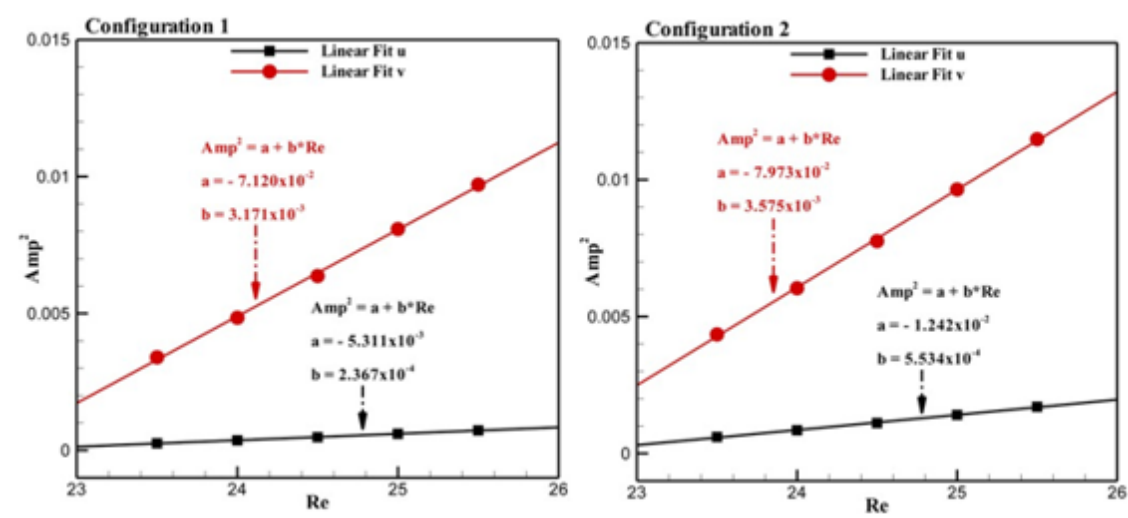

Figure 9: Variation number for the two configurations of the square amplitude of the oscillations of $u$ and $v$ velocity components at the same point as a function of the Reynolds.

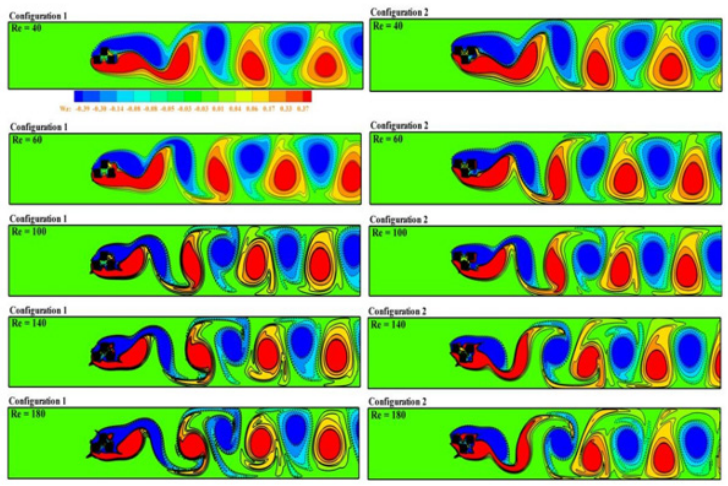

Figure 10: Vorticity contour plots of flow at different Reynolds numbers relatively to both considered arrangements.

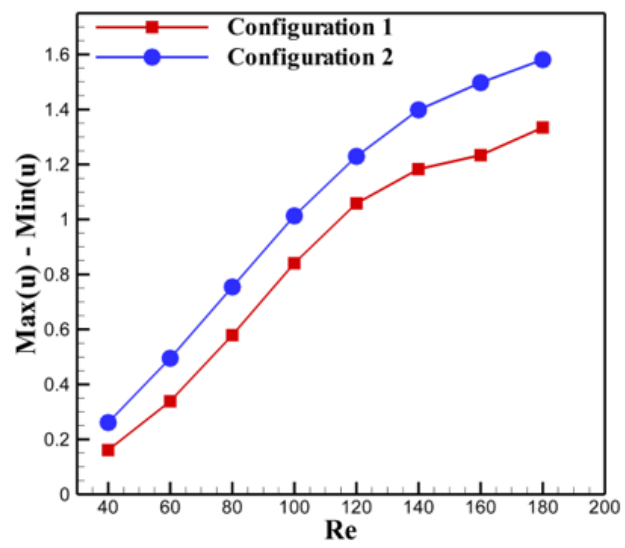

Figure 11: Variation (Max (u) -Min (u)) as a function of Re. 


\section{Analysis of the fluctuation of Strouhal number}

In this study, a fast Fourier transform of the time series for the lift coefficients is used to determine the Strouhal number. It can be seen in Figure 12 that the Strouhal number of the two configurations increases monotonously with Rewhen $30 \leq \mathrm{Re} \leq 80$. This depends on the inertial force which strengthens with the increase of $\operatorname{Re}$. When $30 \leq \operatorname{Re} \leq 80$, it is noted that the values of the frequency of detachment of the vortices are very close in both configurations. It is clear that the obstacles geometry has no effect on the evolution of the Strouhal number when the Reynolds number slightly varies. Moreover, we find that the first configurations reach the maximum before the second configuration. For the first configuration, this maximum is in the vicinity of a Reynolds which is equal to 90 . On the other hand, for the second configuration, this maximum value was noted for a Reynolds number very close to 100 (Figure 12).

When $R e \geq 90$, a considerable change in the fluctuation of the Strouhal number in both configurations is observed and at this stage appears the effect of the geometry and the symmetry of the configurations on the fluctuation of the Strouhal number. The values of St in the second configuration become greater than the values of St in the first configuration. This change can be due to the influence of the geometry of the obstacle on the detachment of the vortices. The first configuration hence generates a reduction in the fluctuation of St compared with the second one, and this reduction becomes very important for higher values of the Reynolds number.

Figure 13 illustrates a comparison between the values of St of both configurations with the case of a square cylinder. It is observed that the two configurations generate a significant reduction in the values of the Strouhal number compared with the square cylinder case. It is clear that this reduction becomes more significant with Re and seems to be very important when the Reynolds number has a value 160 . The percentage of reduction of St for both configurations was determined in Table 4. We find that for low Reynolds numbers $(\mathrm{Re}=60$ and 80$)$ the best reduction is caused by the second configuration and the lowest reduction corresponds to the first one. Starting from the value $\mathrm{Re}=100$, the percentage of reduction augments in the first configuration and becomes the highest in this configuration compared to the other one. Note that the maximum reduction percentage in the first arrangement and the second one is respectively $29.10 \%$ (when $\mathrm{Re}=140$ ) and $27.90 \%$ (when $\mathrm{Re}=$ 160) (Table 4).

\section{Analysis of the fluctuation of the average drag coefficient}

The drag coefficient corresponds to the force applied to the body in the flow direction which is made dimensionless by the density of the fluid. Its velocity at upstream direction $\left(U_{\infty}\right)$ is a characteristic surface calculated from the side of the shape and its size.

The drag coefficient is computed as follows:

$$
C D=\frac{F_{D}}{\frac{1}{2} \rho U_{\infty}^{2} H}=C D_{P}+C D_{V}
$$

Where $\mathrm{CD}_{\mathrm{P}}$ and $C \mathrm{D}_{\mathrm{V}}$ represent the drag coefficients due to pressure and viscous forces. $F_{D}$ is the drag force acting on the obstacle surfaces. Thus, the drag coefficient due to viscous and pressure forces can be obtained from the following expressions:

$$
C D=2 \int_{0}^{1}\left(P_{f}-P_{r}\right) d y+\frac{2}{\operatorname{Re}} \int_{0}^{1}\left\{\left(\left(\frac{\partial u}{\partial y}\right)_{t}+\left(\frac{\partial u}{\partial y}\right)_{b}\right) d x+\left(\left(\frac{\partial u}{\partial x}\right)_{f}+\left(\frac{\partial u}{\partial x}\right)_{r}\right) d y\right\}
$$

The subscripts $\mathrm{f}, \mathrm{r}, \mathrm{b}$ and $\mathrm{t}$ refer to the front, rear, bottom and top surfaces of the obstacle.

The average values of the drag coefficient of the two configurations when Re varies from 1 to 180 are reported in Table 5 (Table $5)$.

Table 5 shows that the average drag coefficient (CD) of the two configurations has a fairly high value at $\mathrm{Re}=1$ since the velocity seems to be much weakened and hence a higher pressure is established. Thus, we may predict the higher value of CD in the first configuration and the lowest one in the second configuration. It can then be concluded that the first configuration generates a pressure rise when compared with the second one. When the Reynolds number is further raised, a sharp decrease is noticed in the fluctuation of the average drag coefficient and observed for both configurations. This decrease is related to the growth of the intensity of the forces of inertia and the lowering of the intensity of the viscous ones. In addition, it can be seen that when the flow remains steady $(\operatorname{Re}=1-10-20)$, the highest values of $C D$ are noted in the first configuration and the lowest values are obtained for the second configuration. The diminution in CD can be explained by the velocity strengthening as shown in Figure 12 and therefore the decrease in pressure because the change in pressure is inversely proportional to the speed variation. By further enhancing the Reynolds number, a decrease in the CD values is seen and this leads to lessening pressure and strengthening the speed (Figure 11). It should be noted that from a $\mathrm{Re}=30$ the $\mathrm{CD}$ values are very close in both configurations. Consequently, the influence of the geometry of the obstacle on the variation of the drag force strengthens and becomes significant while lowering the Reynolds number. However, the effect of the geometry decreases while increasing the Reynolds number. In the range $60 \leq \operatorname{Re} \leq 180$, the flow undergoes an unsteady behavior. The highest CD values are then noted in the second configuration. This is due to the enhancement in the intensity of the forces applied by the flow on this obstacle and to the decrease in pressure which becomes almost negligible. Conversely at steady state where the pressure forces are very high and the velocity variation is very low, $C D$ values become very low in the first configuration when the flow state is unsteady. 
Figure 14 is plotted in order to compare the values of the average drag coefficient of the two configurations considered in this study with the CD values of a square cylinder. It can be seen that the shape of the two configurations considerably modifies the variation of pressure and velocity in the flow. This results in a change in the fluctuation of the drag force. Therefore, it can be observed that the CD values noted in the flow around a square cylinder are very small compared to the values of the two configurations. It can be deduced then that the two configurations generate an enhancement in the intensity of the drag force compared to the case of a square cylinder.

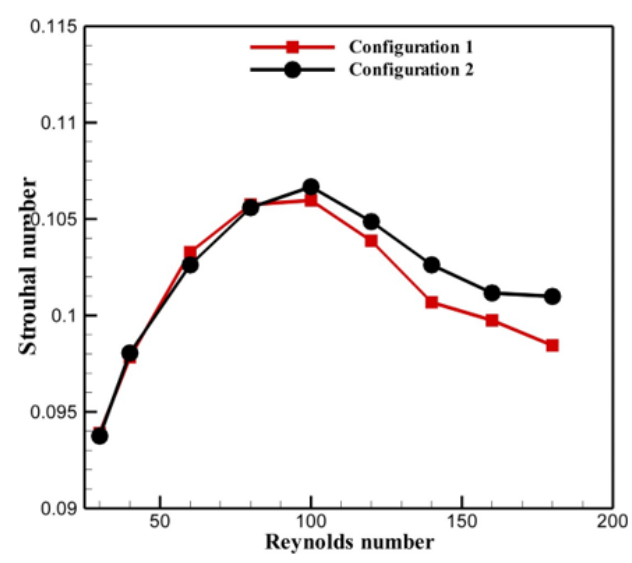

Figure 12: Variation of St as a function of Re for both considered arrangements.

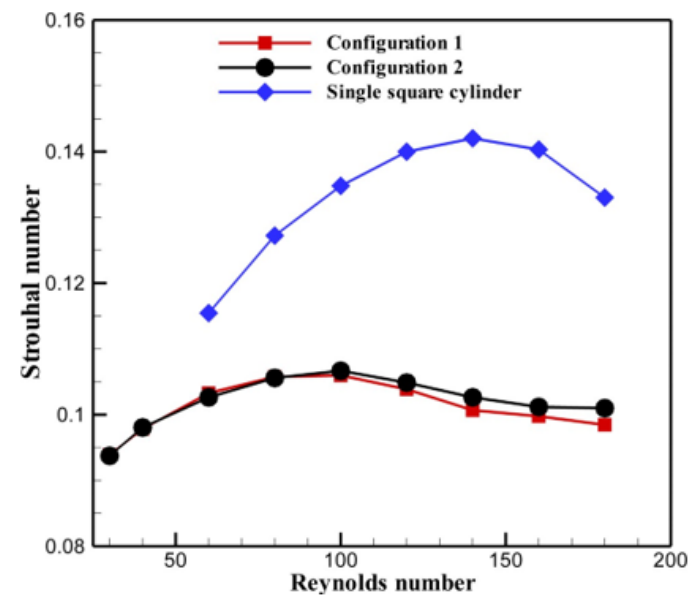

Figure 13: Strouhal number as a function of Reynolds number relatively to the considered configurations.

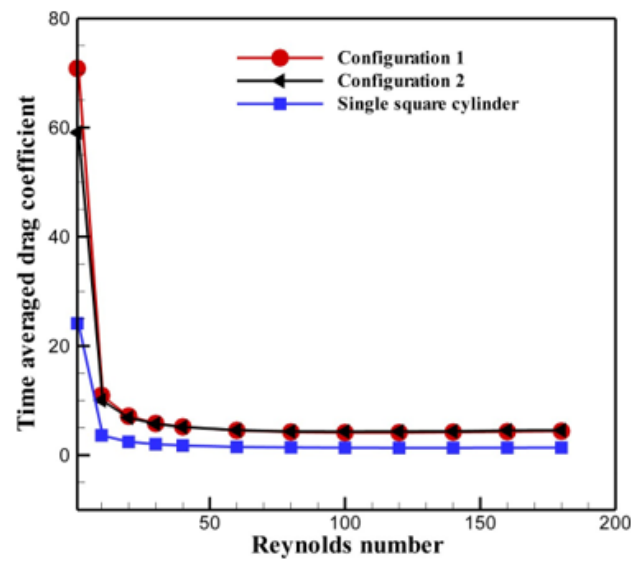

Figure 14: Time-averaged drag coefficient evolution as a function of Reynolds number for the two configurations. 


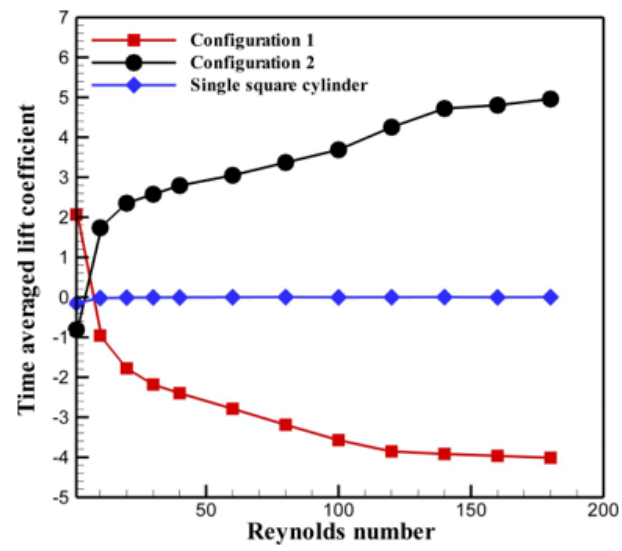

Figure 15: Time averaged lift coefficient evolution as a function of Reynolds number for the two configurations.

\section{Analysis of the fluctuation of the average lift coefficient}

The lift coefficient corresponds to the force applied to the body perpendicular to the incident flow, which is made dimensionless by

the density of the fluid. Its velocity at upstream direction $\left(U_{\infty}\right)$ is a characteristic surface calculated from the side of the shape and its size.

The lift coefficient is computed from:

$$
C L=\frac{F_{L}}{\frac{1}{2} \rho U_{\infty}^{2} H}=C L_{P}+C L_{V}
$$

Where $\mathrm{CL}_{\mathrm{P}}$ and $\mathrm{CL}_{\mathrm{V}}$ represent the lift coefficients due to pressure and viscous forces respectively. $\mathrm{F}_{\mathrm{L}}$ is the lift force acting on the obstacle surfaces. Thus, the lift coefficient due to viscous and pressure forces can be obtained from the following expressions:

$$
C L=2 \int_{0}^{1}\left(P_{b}-P_{t}\right) d x+\frac{2}{\operatorname{Re}} \int_{0}^{1}\left\{\left(\left(\frac{\partial v}{\partial x}\right)_{f}+\left(\frac{\partial v}{\partial x}\right)_{r}\right) d y+\left(\left(\frac{\partial u}{\partial x}\right)_{t}+\left(\frac{\partial u}{\partial x}\right)_{b}\right) d x\right\}
$$

The subscripts $\mathrm{f}, \mathrm{r}, \mathrm{b}$ and $\mathrm{t}$ refer to the front, rear, bottom and top surfaces of the shape.

To understand the link between the fluctuation of the lift force and the shape of the obstacle, we present the figure 15. This figure shows the variation of the time average value of the lift coefficient versus the Reynolds number for both configurations. First, it is noted that the largest value of CL corresponds to the first configuration and the lowest value is related to the second one. As the Reynolds number augments, the curve relative to the second configuration exhibits a rising trend. By cons, in the first configuration the average value of the lift coefficient lowers. It is clear that the curves of the two configurations evolve symmetrically with respect to the curve of the square configuration. This indicates that the direction of the lift force applied to the first configuration is opposite to the direction of the lift force of the second configuration. Therefore, this reversal evolution in the direction of the lift force is due to the shape of both configurations. When $10 \leq \mathrm{Re} \leq 180$, the average lift coefficients of the first configuration are negative, while those of the second configuration are positive. It can be seen then that the geometry of the obstacle considerably affects the fluctuation of the lift force. Indeed, it can be observed that the first configuration generates a large reduction in the average lift coefficient compared with the square configuration. However, the second configuration generates a considerable enhancement in the values of the average lift coefficient (Figure 15).

\section{Conclusion}

The flow patterns past three square cylinders at two symmetrical vee shapes have been numerically performed for $1 \leq \operatorname{Re} \leq$ 180. Two different arrangements were considered and compared in terms of flow behavior and characteristics. An accelerated multigrid implicit volume method was used as a numerical technique for the present study. Firstly, the computations were carried out for flow behind a single square cylinder in order to check the validity and accuracy of the in-house NASIM code. Then, the flow characteristics of both considered arrangements were investigated in terms of vorticity contour visualization, time evolutions analysis of drag and lift coefficients. Main findings are summarized below:

1. It is found that both the arrangements and the geometry of the obstacle affect the flow characteristics significantly as well as the appearance of bifurcation point from steady to the unsteady state. Indeed, it is seen that the transition to the unsteady state begins in the second configuration from $(\mathrm{Rec}=22.37)$ and then emerge in the first configuration precisely at the value $(\operatorname{Rec}=$ 22.45). Besides, both configurations considered in this study generate an important decline in the critical Reynolds number value and accelerate the birth and vortices generation.

2. Influence of the obstacle geometry on the vortex detachment and the Strouhal number variation versus Re have been analyzed and discussed for the two configurations. It is seen that 
varying the arrangements leads to a considerable modification in the fluctuation of the Strouhal number.

3. In addition, fluid behavior of both arrangements were analyzed and compared with single cylinder case. The numerical results show that the two configurations generate a significant reduction in the values of the Strouhal number compared with the square cylinder. This reduction reinforces with increment in Re and becomes more significant when the Reynolds number reaches the value 160 .

4. When analyzing the effect of the obstacle geometry on the flow structure and the frequency of vortices detachment, the results also suggest that both arrangements considerably modify the variation of pressure and velocity in the flow. This results in a variation in the typical fluctuations of the drag force.

5. Considering the geometry influence of both configurations on the lift, it is observed that the geometry of the obstacle noticeably affects the fluctuation of the lift force with symmetrical evolutions for both configurations. This reversal distribution trend demonstrates that the direction of the lift force applied to the first configuration is opposite to the lift force direction of the second one due to the shape of both of them.

\section{Acknowledgement}

None.

\section{Conflicts of Interest}

No conflict of interest.

\section{References}

1. Cheng M, Whyte DS, Lou J (2007) Numerical simulation of flow around a square cylinder in uniform- shear flow. J Fluids Struct 23(2): 207-226.

2. Berrone S, Garbero V, Marro M (2011) Numerical simulation of lowReynolds number flows past rectangular cylinders based on adaptive finite element and finite volume methods. Computers \& Fluids 40(1): 92-112.

3. Mukhopadhyay A, Biswas G, Sundararajan T (1992) Numerical investigation of confined wakes behind a square cylinder in a channel. Int J Numer Meth Fluids 14: 1473-1484.

4. Fezai S, Ben CheikhN, Ben-Beya B, Lili T (2016) Obstacle geometry effect on the stability of two- dimensional incompressible flow in a channel. JAFM 9: 625-633.

5. Lankadasu A,Vengadesan S (2008) Onset of vortex shedding in planar shear flow past a square cylinder. Int. J. Heat and Fluid Flow 29: 10541059.

6. Sohankar A, Etiminan A (2009) Forced convection heat transfer from tandem square cylinders in cross flow at low Reynolds numbers, international journal for numerical methods in fluids 60: 735-751.

7. Gera B, Pavan K Sharma, Singh RK (2010) CFD analysis of 2D unsteady flow around a square cylinder. Int. J. Appl. Eng. Res. DINDIGUL 1: 602610.

8. Bhattacharyya S, Maiti DK (2004) Shear flow past a square cylinder near a wall. Int J Eng Sci 42: 2119-2134.

9. Sajjad M, Sohn CH (2014) Numerical study of flow past a square cylinder with corner curvature at incidence. 11th International Bhurban Conference on Applied Sciences and Technology (IBCAST) pp. 294-297.
10. Burattini P, Agrawal A (2013) Wake interaction between two side-byside squares cylinders in channel flow. Comput Fluids 77: 134-142.

11. Abbasi WS, Islam SU (2018) Transition from steady to unsteady state flow around two inline cylinders under the effect of Reynolds numbers. J Braz Soc Mech Sci Technol 40(168): 1-12.

12. Rao Y, Ni Y, Liu C (2008) Flow effect around two square cylinders arranged side by side using Lattice Boltzmann method. Int. J. Mod. Phys. C 11: 1683-1694.

13. E Adeeb, Haider BA, Sohn GH (2018) Flow interference of two side-byside square cylinders using IB-LBM- effect of corner radiuns. Results in Physics 10: 256-263.

14. Lee K, Yang KS (2009) Flow patterns past two circular cylinders in proximity. Computers \& Fluids 38(4): 778-788.

15. Aboueian J, Sohankar A (2017) Identification of flow regimes around two staggered square cylinders by a numerical study. Theor Comput Fluid Dyn.

16. Islam SU, Abbasi WS, Zhou CY (2016) Transitions in the unsteady wakes and aerodynamic characteristics of the flow past three square cylinders aligned inline. Aerospace Science and Technology 50: 96-111.

17. Islam SU, Rahman H, Zhou CY (2016) Effect of separation ratios on flow past row of rectangular cylinders with aspect ratio 1.5. Ocean Engineering 119: 1-15.

18. H Rahman, SU Islam, WS Abbasi, G Nazeer (2020) A numerical study for flow around three square cylinders in triangular arrangement. Iranian Journal of Science and Technology, Transactions of Mechanical Engineering. 44: 229-246.

19.S Yang, W Yan, J Wu, C Tu, D Luo (2016) Numerical investigation of vortex suppression regions for three staggered circular cylinders. Eur J Mech B Fluids 55: 207-214.

20.W Yan, J Wu, S Yang, Y Wang (2016) Numerical investigation on characteristic flow regions for three staggered stationary circular cylinders. Eur. J. Mech. B Fluids 60: 48-61.

21. Sohankar A, Norberg C, Davidson L (1998) Low Reynolds number flow around a square cylinder at incidence: Study of blockage, onset of vortex shedding and outlet boundary condition. Int J Numer Meth Fluids 26 : 39- 56.

22. Hortmann M, Peric M,Scheuerer G (1990) Finite volume multigrid prediction of solutions laminar natural convection: Bench-Mark. Int J Numer Meth Fluids 11: 189-207.

23. Brown DL, Cortez R, Minion ML (2001) Accurate projection methods for the incompressible Navier-Stokes equations. Comput Mech 168: 464-499.

24. Hayase T, Humphrey JAC, Greif R (1992) A consistently formulated QUICK scheme for fast and stable convergence using finite-volume iterative calculation procedures. J Comput Phys 98: 108-118.

25. Hadjidimos A (2000) Successive overrelaxation (SOR) and related methods. J Comp App Math 123:177-199.

26. Fezai S, Ben Cheikh N, Ben Beya B, Lili T (2017) Numerical study of Vortex shedding suppression and aerodynamic characteristics of three obstacle configurations having two shapes. J Braz Soc Mech Sci Technol 39: 2519-2534.

27. Ben Beya B, Lili T (2008) Three-dimensional incompressible flow in a two-sided non-facing lid-driven cubical cavity. CR Mecanique 336: 863872 .

28. Fezai S, Oueslati F, Ben-Cheikh N, Ben-Beya B (2018) Sensitivity of wake parameters to diameter changes for a circular cylinder. Int J Mod Phys C29 pp.1850087.

29. Breuer M, Bernsdorf J, Zeiser T, Durst F (2000) Accurate computations of the laminar flow past a square cylinder based on two different methods: lattice-Boltzmann and finite-volume. Int. J. Heat and Fluid Flow 21: 186196. 
30. Galletti B, Bruneau CH, Zannetti L, Iollo (2004) A Low-order modelling of laminar flow regimes past a confined square cylinder. J Fluid Mech 503:161-170.

31. Fezai S, Ben Cheikh N, Ben-Beya B, Lili T (2019) Numerical study of obstacle geometry effect on the vortex shedding suppression and aerodynamic characteristics. Int J Numer Meth Heat Fluid Flow.
32. Fezai S, Oueslati F, Ben-Cheikh N, Ben-Beya B (2019) Prediction of wake structure and aerodynamic characteristics of flow around square cylinders at different arrangements. Int J Mod Phys C 30, pp.1950015. 